\title{
Editorial
}

\section{Interoception and Health}

\section{Psychological and Physiological Mechanisms}

\author{
Beate M. Herbert ${ }^{1,2}$, Olga Pollatos ${ }^{3}$, and Verena Klusmann ${ }^{4,5}$ \\ ${ }^{1}$ Clinical Psychology \& Psychotherapy, Department of Psychology, Eberhard-Karls-University of Tübingen, Germany \\ 2 Psychology School, University of Applied Sciences Fresenius, Munich, Germany \\ ${ }^{3}$ Health \& Clinical Psychology, Institute of Psychology and Education, Ulm University, Germany \\ ${ }^{4}$ Department of Psychology, Psychological Assessment \& Health Psychology, University of Konstanz, Germany \\ ${ }^{5}$ Department of Psychology and Human Movement Science, University of Hamburg, Germany
}

The present special issue of the European Journal of Health Psychology (EJHP) is dedicated to the topic of interoception and its importance for health and disease. The four contributions, two conceptual articles, and two original articles, go back to a symposium (organized by Beate Herbert) at the conference of the Health Psychology Section (Fachgruppe Gesundheitspsychologie) of the German Society of Psychology (Deutsche Gesellschaft für Psychologie e.V.) in 2019 and were subsequently invited for a special issue on the initiative of the editorial board of the EJHP.

Actual research and models of "embodied" or "grounded" cognition highlight that our mental functions and knowledge about the world are fundamentally grounded in the body and the body's interaction with the environment. Awareness and experience of ourselves and the external world are based on multisensory integrations across sensory modalities. The importance of the sense of ourselves from within for understanding adaptive behavior, health, and psychopathology has been increasingly recognized during the last decades in psychological and neuroscientific research. Scientific interest in interoception has fluctuated during the years since Charles Sherrington's first usage of the concept in 1906. Its "intrinsic" relevance dates back to important scientific milestones like Darwin's "expression of emotion" (1872), Claude Bernard's "milieu interior" (1878), or Williams James' "principles of psychology" (1890). The recent years witnessed a surge of interest in the topic of interoception, which is due to the "embodiment paradigm shift" in psychology and neurosciences, recognizing that intelligent or adaptive behavior needs a body behaving within contexts, and which is promoted by findings highlighting the integral role of interoception in self- regulation, emotional experience, decision making, and consciousness (Khalsa et al., 2018). Interoception, as the visceral dimension of embodiment (Herbert \& Pollatos, 2012; Pollatos \& Herbert, 2018; Quadt et al., 2018), has gained rapidly expanding interest in the study of the human mind, especially stimulated by the seminal work of Bud Craig (2009) and Antonio Damasio (1999), who laid the scientific foundation of interoceptive processes as the center of self-awareness.

Interoception has been described as the body-to-brain axis of sensations concerning the internal state of the body (Cameron, 2002; Craig, 2009), and refers primarily to the sensing and representation of ascending information from visceral organs (such as cardiovascular, gastrointestinal, respiratory, and hormonal systems). Whether proprioception (signals concerning the skin and musculoskeletal apparatus to control the body in space) should be included in the concept of interoceptive abilities is currently under debate. In the following, we will focus on visceroception, and use the term interoception to describe the processing and perception of signals from the inner organs, that play a major role in ensuring homeostatic and allostatic adaptations of the organism and regulation of its associated needs. Going beyond homeostatic regulation, interoception has been evidenced to be fundamental for dimensions of the "bodily self" and mechanisms of embodied cognition, that is, for shaping cognition, emotion, and behavior. Interoception represents the basis of our experienced body that lies at the core of awareness of our "self," which is considered as an integrative structure of the mind that organizes and coordinates affective, cognitive, social sensorimotor, and vegetative functions. 
Increasing evidence demonstrates the crucial role of interoceptive processes and their corresponding psychological concepts for mental and physical health as well as disease, and dysfunctional interoception has been suggested to represent an important transdiagnostic factor across a range of clinical psychological disorders (e.g., Khalsa et al., 2018; Murphy et al., 2017). The latter range from aberrant emotional processing to anxiety and stress-associated diseases, to maladaptive eating behavior, eating disorders, and obesity, issues, that will be addressed in the present special issue. Furthermore, interoceptive deficits are of relevance in subclinical personality constructs associated with deficits in emotional awareness and dysfunctions of a stable and unified sense of self (see Tsakiris \& De Preester, 2018).

Interoception is a multifaceted construct and different taxonomies have been proposed to differentiate distinguishable and independent interoceptive dimensions. Recent concepts of interoception (e.g., Garfinkel et al., 2015, 2016) primarily differentiate interoceptive accuracy (IAcc), interoceptive sensibility (IS), and interoceptive awareness (IA). IAcc has been defined as the ability to more or less accurately detect or perceive signals from within the body such as one's heartbeats, respiration-related signals, hunger, or fullness. It is estimated by the individual's perception performance in behavioral tests, like heartbeat perception paradigms in the cardiac modality. Interoceptive sensibility (IS) is typically assessed by subjective self-report questionnaires, and the dimension of interoceptive awareness (IA) combines subjective and objective measures aiming to assess metacognitive facets of interoception. A fourth dimension that has been recently addressed is interoceptive evaluation (IE) (Herbert \& Pollatos, 2018; Herbert et al., 2012, 2013; Pollatos \& Herbert, 2018). These and further concepts will be described and discussed in more detail in the contributions by Herbert (2020), as well as MaiLippold and colleagues (2020) in this special issue. Regarding existent research findings on the relevance of interoceptive processes for health and disease, IAcc and facets of self-reported IS are the dimensions that have been most amply investigated. The specific role of further dimensions of interoception, as well as the question, if and how these are related to each other as well as to mechanisms of health and disease has hardly been investigated so far. This is the subject of current research and will be discussed in some contributions of the present special issue.

Neuroscientific evidence highlights that interoceptive abilities are reflected in the interoceptive neural network in the brain, including somatosensory and somatomotor cortices, insular cortex, anterior cingulate cortex, and prefrontal cortices. These are relevant for monitoring the internal emotional and viscerosensory state, for emotion processing and reactivity, and for self-regulation of feelings and behavior (e.g., Critchley \& Garfinkel, 2017; Quadt et al., 2018; Pollatos et al., 2007, 2016). Within this interoceptive network, the insula represents an important projection site of viscerosensory input from different modalities (i.e., different organ systems) from the body. Distinguishable regions of the insula are suggested to be involved in different and successive steps of neural processing. This builds the basis for the sequential integration of the primary homeostatic condition of the body with salient features of the sensory environment and with hedonic, motivational, and social conditions (e.g., Barrett \& Simmons, 2015; Quadt et al., 2018; Seth \& Friston, 2016). This network in the brain may be seen as the neuroanatomic substrate for conceptualizing interoception as the basis of the "material me" (Craig, 2009).

Actual neuroscientific Bayesian theories of the brain have developed to understand interoception within the scope of predictive coding models suggesting that interoceptive experience reflects predictions about the expected state of the body that are constrained by ascending visceral sensations (Barrett \& Simmons, 2015). A "sense of presence" has been suggested to arise when informative interoceptive prediction signals are successfully matched to sensory inputs so that prediction errors are minimized (Seth et al., 2011). Within this framework, interoceptive accuracy (IAcc) may be seen as an estimate of "precision" and/or as an indicator of more "reliable" interoceptive information. Symptoms of clinical conditions that have been amply related to interoceptive dysfunction may be understood as malfunctions within the process of minimizing interoceptive and bodily prediction errors (e.g., Paulus et al., 2019; Quadt et al., 2018).

Grounded on these models and on ongoing empirical research, this special issue addresses the relevance of interoception for health and disease-related processes. The four contributions provide insight into relevant physiological and psychological mechanisms and concepts of interoception, and its role in shaping selected conditions of health and disease:

The first conceptual article by Schulz and colleagues (2020) gives an overview of psychophysiological mechanisms of interoception and reviews relevant findings showing that a dysregulation of the brain-body communication represents an important mechanism for the generation of physical symptoms in stress-related disorders. The authors summarize current knowledge on acute stress effects mediated via the physiological stress axes activities on cardiac interoception, as assessed by behavioral tasks measuring cardiac interoceptive accuracy and the heartbeat evoked potential, representing a valid brain correlate of cardiac interoception. The authors discuss possible patterns of abnormal interoception and stress processes in mental disorders and chronic conditions as well as techniques to change interoception. 
In the second contribution, Nusser and colleagues (2020) present results of an original study on age-related effects of interoceptive accuracy and interoceptive sensibility, as assessed by cardiac IAcc and by scales assessing general and specific IS, in 146 individuals aged between 19 and 81 years. The authors investigated if earlier findings showing a decrease of interoceptive accuracy across the adult lifespan are replicated when considering a greater sample size as well as by using statistical ameliorations, and by including measures of IS. Different age effects on the three dissociable interoceptive dimensions were found: The results support that IAcc decreases with age, but specific IS tend to increase with age and general IS appear to remain unaffected by age. Also, specific and general IS exhibited different relationships with cardiac IAcc. These findings are discussed with respect to age-related changes in neurophysiological and psychological functions, as well as with regard to the meaning of heterogeneous changes of IAcc and IS with age, and the emergence of physical diseases.

In the third contribution, Mai-Lippold and colleagues (2020) examine the effects of energy drinks on cardiac IAcc, IS, and IE, including cortical processing represented by the heartbeat-evoked potential (HEP), in an original study of healthy participants varying in anxiety sensitivity levels. Their results reveal no effects of energy drinks on interoception. However, participants with high levels of anxiety sensitivity reported significantly reduced interoceptive confidence after energy drink consumption as well as reduced HEPs at seven of eight investigated clusters as compared to subjects with low anxiety sensitivity. The authors discuss their findings of lower confidence in perceived interoceptive abilities in subjects with high anxiety sensitivity as an effect of higher uncertainty regarding the classification of internal bodily signals, and/or as a misinterpretation of those signals accompanied by an immediate fear of loss of control over bodily and mental processes, as has been shown in patient groups with anxiety disorders. The findings are interpreted as being indicative of a greater prediction error in the high anxiety sensitivity group.

The fourth contribution, the conceptual article by Herbert (Herbert, 2020) provides an overview of research findings and concepts of interoception and its relevance for adaptive and maladaptive eating behavior, obesity, and eating disorders. Going beyond eating in its narrow sense, that is intrinsically guided by interoceptive signals and closely associated with homeostatic psychophysiological needs, survival, and well-being, eating disorders are characterized as disorders of the embodied self, with dysfunctional interoception at its core. Herbert delineates dissociable dimensions and constructs of interoception, provides empirically and conceptually drawn conclusions on the role of interoception in shaping eating behavior, and conceptualizes eating disorders within a framework of predictive coding models. Predictive coding accounts are addressed to integrate conclusions and to classify and underline the relevance of interventions to modify interoception.

The collected contributions on selected topics discussing the role of interoception for health and disease are far from being exhaustive, but we feel that they give an aptly and compact overview. The contributions highlight the importance of understanding interoceptive processes in shaping mental and behavioral functions and the authors discuss mechanisms and models within the actual scientific discourse of interoception research.

Looking back on a turbulent year in times of COVID-19, which not only was demanding both in private, educational, and research regards, but also provided us with important new scientific insights, one thing is clear: The pandemic is a biopsychosocial challenge - it requires our basic and applied scientific expertise as health psychologists. In this sense, we thank you for your interest and support of the EJHP and look forward to being a European and international platform for scientific exchange on current health psychology topics also in the upcoming year. We hope you enjoy reading the special issue.

With kind regards and best wishes from all EJHP editors,

Ulm/Hamburg, December 2020

Beate M. Herbert, Olga Pollatos, Verena Klusmann

\section{References}

Barrett, L., \& Simmons, W. K. (2015). Interoceptive predictions in the brain. Nature Reviews Neuroscience, 16(7), 419-429. https://doi.org/10.1038/nrn3950

Bernard, C. (1878). Leçons sur les phénomènes de la vie communs aux animaux et aux végétaux [Lectures on the phenomena of life common to animals and plants]. B. Baillière et fils.

Cameron, O. (2002). Visceral sensory neuroscience: Interoception. Oxford University Press.

Craig, A. D. B. (2009). How do you feel - now? The anterior insula and human awareness. Nature Reviews: Neuroscience, 10(1), 59-70. https://doi.org/10.1038/nrn2555

Critchley, H., \& Garfinkel, S. (2017). Interoception and emotion. Current Opinion in Psychology, 17, 7-14. https://doi.org/ 10.1016/j.copsyc.2017.04.020

Damasio, A. (1999). The feeling of what happens: Body, emotion and the making of consciousness. Harcourt.

Darwin, C. (1872). The expression of the emotions in man and animals. John Murray.

Garfinkel, S. N., Seth, A. K., Barrett, A. B., Suzuki, K., \& Critchley, H. D. (2015). Knowing your own heart: Distinguishing interoceptive accuracy from interoceptive awareness. Biological Psychology, 104, 65-74. https://doi.org/10.1016/j.biopsycho.2014.11.004

Garfinkel, S. N., Tiley, C., O'Keeffe, S., Harrison, N. A., Seth, A. K., \& Critchley, H. D. (2016). Discrepancies between dimensions of 
interoception in autism: Implications for emotion and anxiety. Biological Psychology, 114, 117-126. https://doi.org/10.1016/j. biopsycho.2015.12.003

Herbert, B. M. (2020). Interoception and its role for eating, obesity, and eating disorders: Empirical findings and conceptual conclusions. European Journal of Health Psychology, 27(4), 188-205. https://doi.org/10.1027/2512-8442/a000062

Herbert, B. M., Blechert, J., Hautzinger, M., Matthias, E., \& Herbert, C. (2013). Intuitive eating is associated with interoceptive sensitivity. Effects on body mass index. Appetite, 70, 22-30. https://doi.org/10.1016/j.appet.2013.06.082

Herbert, B. M., Herbert, C., Pollatos, O., Weimer, K., Enck, P., Sauer, H., \& Zipfel, S. (2012). Effects of short-term food deprivation on interoceptive awareness, feelings and autonomic cardiac activity. Biological Psychology, 89(1), 71-79. https://doi.org/10.1016/j.biopsycho.2011.09.004

Herbert, B. M., \& Pollatos, O. (2012). The body in the mind: On the relationship between interoception and embodiment. Topics in Cognitive Science, 4(4), 692-704. https://doi.org/10.1111/ j.1756-8765.2012.01189.x

Herbert, B. M., \& Pollatos, O. (2018). The relevance of interoception for eating behavior and eating disorders. In M. Tsakiris \& H. De Preester (Eds.), The interoceptive mind (pp. 165-186). Oxford University Press. https://doi.org/10.1093/oso/9780198811930.003. 0009

James, W. (1890). The principles of psychology. H. Holt and Company.

Khalsa, S. S., Adolphs, R., Cameron, O. G., Critchley, H. D., Davenport, P. W., Feinstein, J. S., Feusner, J. D., Garfinkel, S. N., Lane, R. D., Mehling, W. E., Meuret, A. E., Nemeroff, C. B., Oppenheimer, S., Petzschner, F. H., Pollatos, O., Rhudy, J. L., Schramm, L. P., Simmons, W. K., Stein, M. B., ... Zucker, N. (2018). Interoception and mental health: A roadmap. Biological Psychiatry: Cognitive Neuroscience and Neuroimaging, 3(6), 501-513. https://doi.org/10.1016/j.bpsc.2017.12.004

Mai-Lippold, S., Dettlinger, C. M., Khalsa, S., \& Pollatos, O. (2020). A pilot study on the effect of an energy drink on interoception in high vs. low anxiety sensitivity individuals. European Journal of Health Psychology, 27(4), 171-187. https://doi.org/10.1027/ 2512-8442/a000061

Murphy, J., Brewer, R., Catmur, C., \& Bird, G. (2017). Interoception and psychopathology: A developmental neuroscience perspective. Developmental Cognitive Neuroscience, 23, 45-56. https://doi.org/10.1016/j.dcn.2016.12.006

Nusser, L., Pollatos, O., \& Zimprich, D. (2020). Age-related effects on interoceptive accuracy, general interoceptive sensibility, and specific interoceptive sensibility. European Journal of Health Psychology, 27(4), 154-170. https://doi.org/10.1027/2512$8442 / a 000060$

Paulus, P., Feinstein, J. S., \& Khalsa, S. (2019). An active inference approach to interoceptive psychopathology. Annual Reviews of Clinical Psychology, 15, 97-122. https://doi.org/10.1146/ annurev-clinpsy-050718-095617

Pollatos, O., Gramann, K., \& Schandry, R. (2007). Neural systems connecting interoceptive awareness and feelings. Human Brain Mapping, 28(1), 9-18. https://doi.org/10.1002/hbm.20258

Pollatos, O., \& Herbert, B. M. (2018). Interoception: Definitions, dimensions, neural substrates. In G. Hauke \& A. Kritikos (Eds.), Embodiment in psychotherapy: A practitioner's guide (pp. 15-28). Springer. https://doi.org/10.1007/978-3-319-92889-0

Pollatos, O., Herbert, B. M., Mai, S., \& Kammer, T. (2016). Changes in interoceptive processes following brain stimulation. Philosophical Transactions of the Royal Society B: Biological Sciences, 371(1708), Article 20160016. https://doi.org/ $10.1098 /$ rstb. 2016.0016
Quadt, L., Critchley, H. D., \& Garfinkel, S. N. (2018). The neurobiology of interoception in health and disease. Annals of the New York Academy of Sciences, 1428, 112-128. https://doi.org/ 10.1111/nyas.13915

Schulz, A., Schultchen, D., \& Vögele, C. (2020). Interoception, stress, and physical symptoms in stress-associated diseases. European Journal of Health Psychology, 27(4), 132-153. https://doi.org/10.1027/2512-8442/a000063

Seth, A. K., \& Friston, K. J. (2016). Active interoceptive inference and the emotional brain. Philosophical Transaction of the Royal Society B, Biological Sciences, 371, Article 20160007. https://doi.org/10.1098/rstb.2016.0007

Seth, A. K., Suzuki, K., \& Critchley, H. (2011). An interoceptive predictive coding model of conscious presence. Frontiers in Psychology, 2(391), Article 395. https://doi.org/10.3389/fpsyg. 2011.00395

Sherrington, C. S. (1906). The integrative action of the nervous system. Yale University Press.

Tsakiris, M. \& De Preester, H. (Eds.). (2018). The interoceptive mind. Oxford University Press.

Published online January 14, 2021

\section{Beate M. Herbert}

Fabristraße 2

$89075 \mathrm{Ulm}$

Germany

beate.herbert@gmx.de; b.herbert@uni-tuebingen.de

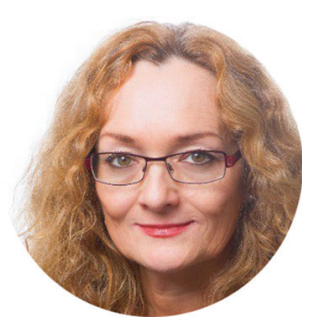

Beate M. Herbert (PhD) is Associate Professor (PD) at the Department of Clinical Psychology and Psychotherapy at Eberhard-Karls-University Tübingen since 2013, and Professor of Biological Psychology and Health Psychology at University of Applied Sciences Fresenius Munich since 2017. In 2016, she founded the Embodiment Research Lab. Her research focuses on psychophysiological mechanisms of interoception and "embodied cognition" in shaping emotional awareness and behavior, and she examines health promotion and interventions based on multimodal body perception and interoception.

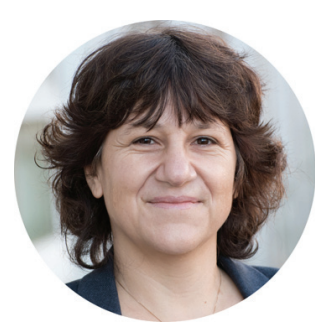

Olga Pollatos (PhD, MD, psychological psychotherapist) is head of Clinical and Health Psychology at the Institute of Psychology and Education at UIm University since 2012. She is also vice president of education at Ulm University since 2018 and chairwoman of the supervisory board of the Baden-Württemberg Centre for Teaching and Learning since 2020. Her research topics include embodied cognition, interoception, emotions and emotion regulation, neurostimulation, stress, health-related interventions, and psychopathological phenomena across the life span. 


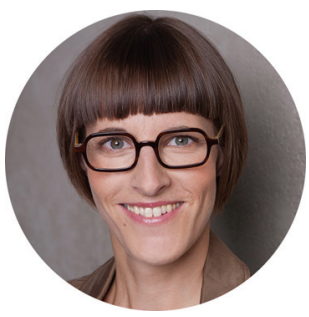

Verena Klusmann $(\mathrm{PhD})$ is chair of the Public Health/Health Sciences Division of the Department of Psychology and Human Movement Science at Hamburg University since 2018. She is also associated with the Division of Psychological Assessment and Health Psychology at University of Konstanz since 2011 and Head of the DFG Scientific Network Images of Aging since 2017. In her research, she examines the dynamics in social cognitions and health behavior changes across the lifespan with a particular focus on health promotion and interventions and on the role of stereotypes and self-perceptions of aging. 